\title{
STRUCTURAL RELATION OF PERIDININ-CHLOROPHYLL $A$ - PROTEIN (PCP) AND PROTEINS WITH GLOBIN-LIKE FOLD
}

\author{
A TESTABLE HYPOTHESIS FOR ITS FOLDING AND ASSEMBLY \\ PATHWAY
}

\author{
KAY DIEDERICHS* and ECKHARD HOFMANN \\ Universität Konstanz, Fakultät für Biologie \\ Postfach 5560 (M656), D-78434 Konstanz, Germany
}

Konstanzer Online-Publikations-System (KOPS)

URN: http://nbn-resolving.de/urn:nbn:de:bsz:352-211652
Abstract: Peridinin-Chlorophyll $a$-Protein (PCP) is a water-soluble, yet membraneattached photosynthetic light-harvesting complex believed to reside in the interior of the thylakoid. We have recently reported the $\mathrm{x}$-ray crystallographic structure of PCP from the dinoflagellate Amphidinium carterae, which crystallizes as a trimer [1]. Overall, the PCP polypeptide ( 312 amino acid residues) has the shape of the hull of a ship enclosing a cargo of two lipids, two chlorophyll $a$ and eight carotenoid molecules. Each monomer is composed of a pseudosymmetry-related $\mathrm{N}$ - and $\mathrm{C}$-terminal half of eight helices each adopting a peculiar topology whose relation to the globin-like fold is explored in this presentation. Based on the resemblance of folds and available data on myoglobin folding, we discuss a possible folding pathway for the PCP holoprotein which has to assemble during and after passage of the apoprotein through the thylakoid membrane.

\section{Introduction}

Higher plants and algae, as well as photosynthetic bacteria have developed light harvesting systems (LHCs), systems which work as antennae and increase the efficiency of photosynthesis by funneling collected light to the membrane-bound reaction centres. Membrane-bound LHCs have been structurally analyzed in the case of bacteria [2] and higher plants [3], and appear to be functionally similar, but structurally unrelated to water-soluble LHCs. Dinoflagellates are algal protists constituting a major portion of world's plankton. Most dinoflagellate species have a unique pigment-protein complex composed of a $32 \mathrm{kDa}$ polypeptide, and two chlorophyll $a$ and eight carotenoid (peridinin) molecules as chromophores. This complex, peridin-chlorophyll $a$-protein

\footnotetext{
•To whom correspondence should be addressed. email: Kay.Diederichs@uni-konstanz.de
} 
$(\mathrm{PCP})$, exists in a form of half of this size in some dinoflagellate species [4]. In the long variant $\mathrm{PCP}$ species, the $\mathrm{N}$ - and the $\mathrm{C}$-terminal halves of the sequence share a high degree of sequence identity (about 55\%), indicating that they have evolved by gene duplication and fusion. As PCP is encoded in the nucleus, it has to be exported from the cytoplasm into the chloroplast and, as its leader sequence indicates, is exported from there into the thylakoid lumen.

PCP has no sequence or structural similarity to any other protein yet determined. Its unique presence in dinoflagellates and its apparent lack of structural relatives have been interpreted [5] such that PCP might be considered a late evolutionary enhancement of the photosynthetic machinery of dinoflagellates, unrelated to other water-soluble LHCs. Here we explore a structural similarity of PCP with proteins of the ubiquitous globin family, and demonstrate its evolutionary relationship to another group of soluble LHC phycobiliproteins, which occurs in cyanobacteria and red algae.

\section{Structure of PCP}

We have recently solved [1] the $\mathrm{x}$-ray structure of PCP from the dinoflagellate Amphidinium carterae, which has a polypeptide of 312 amino acid residues, showing an internal two-fold pseudosymmetry of the $\mathrm{N}$ - and $\mathrm{C}$-terminal domains (Fig. 1). Each domain consists of eight helices arranged in a peculiar fashion, that resembles a 'jellyroll' often encountered in the topology of $\beta$-sheets [8] and observed for the first time for helices in PCP. The juxtaposition of the $\mathrm{N}$ - and $\mathrm{C}$-terminal domains creates the shape of a ship, whose hull is composed of the helices and whose cargo are the cofactors also showing the two-fold pseudosymmetry. In addition to the chromophores, we discovered by crystallographic means two lipid molecules (digalactosyl diacyl glycerol) which are integral structural components of the holoprotein assembly and whose existence and role in PCP is unknown. Trimers of the holoprotein complex appear to be the photosynthetically active unit, which is expected to stack onto the

\section{Figures on following page}

Figure 1: Archirecture of PCP. The monomer of PCP is shown (grey), together with the chromophores (chlorophyll, green; carotenoids, red) and the lipids (blue). The $\mathrm{N}$-terminal domain (left side) is related to the C-terminal domain (right) by a pseudo two-fold axis (vertical).

Figure 2: Three-dimensional (top) and topological (bottom) similarity of proteins from the globin family (left) and PCP (right). The color-ramp indicates the position along the chain from $\mathrm{N}$-terminus (red) to $\mathrm{C}$ terminus (blue). Ark clam hemoglobin was chosen as a typical representative of the globin family.

Figure 3: Binding of the tetrapyrrole cofactors in PCP (green), globins (red) and phycobiliproteins (purple) after superimposition of the $\mathrm{E}$ and $\mathrm{F}$ helices. The globin (ark clam) apoprotein is also shown (grey). All figures were produced with MOLSCRIPT [6] and RASTER3D [7].
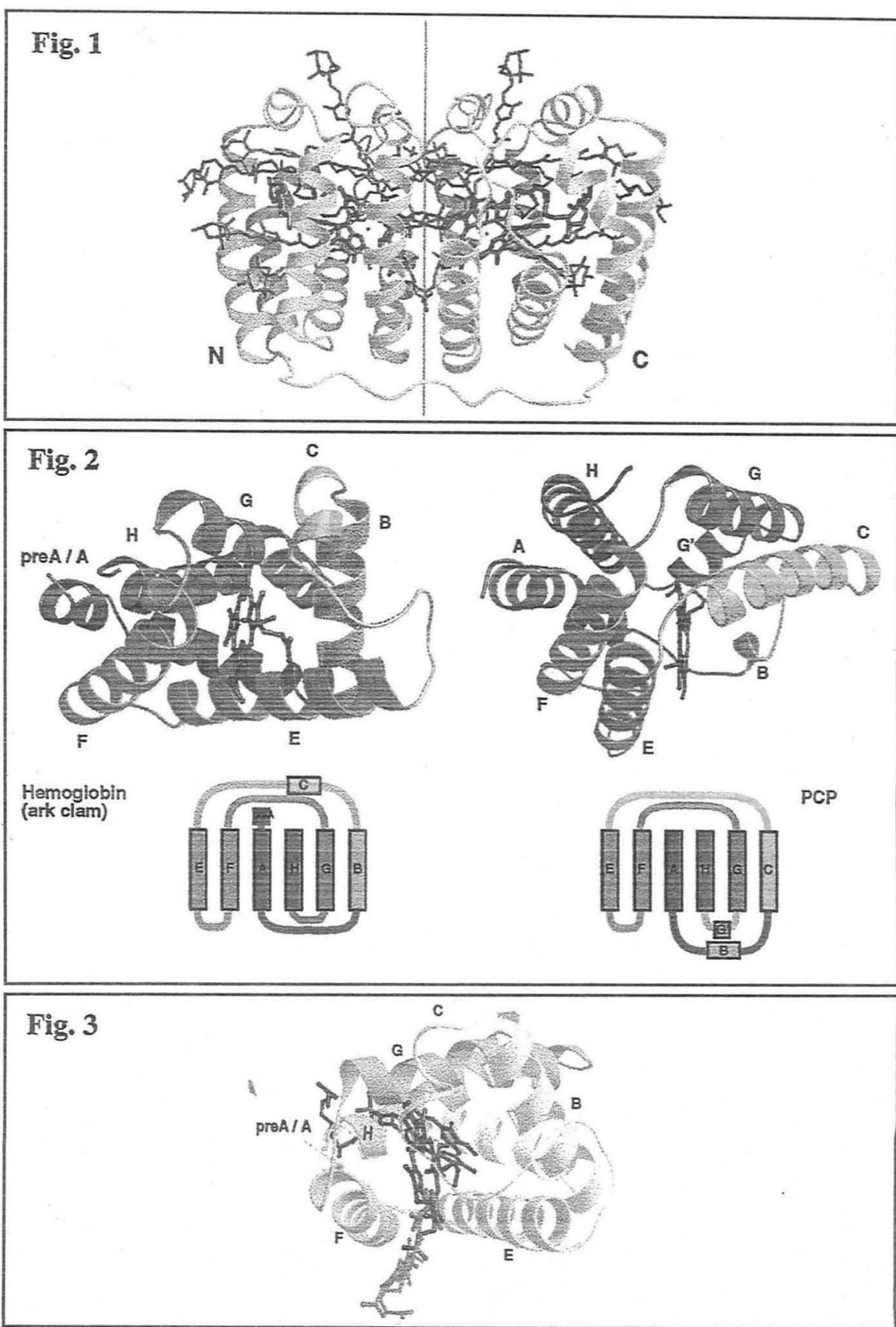
membrane of the thylakoid in order to optimize the transfer of harvested light energy to the membrane-bound components of the photosynthetic apparatus.

Is the existence of two varieties of PCP in dinoflagellates, a large and a small form, compatible with the x-ray structure of PCP from A. carterae? The answer is yes, as the contacts between the domains mainly involve hydrophilic interactions between the peridinin head groups, which could easily be replaced by contacts with an aqueous medium in the case of the short variants. Oligomerization provides a suitable orientation of the chromophores and allows for energy redistribution within the plane of the trimer, thus increasing the efficiency of energy transfer to the membrane-bound photosynthetic proteins. Only the C-terminal domain of the long variants is involved in trimer formation, and its sequence has most likely been optimized for this purpose. Short variant PCPs might form hexamers of the same shape as the long variant's trimers, but these hexamers would certainly be less stable. Thus, the long variant monomers appear to have functional advantages.

\section{Comparison of PCP with the other all- $\alpha$ folds}

Proteins can be considered as evolutionarily related if they share a significant degree of sequence similarity. However, many groups of structurally and functionally related proteins exist that lack similarity detectable with the sequence analysis programs available today [9]. In these groups, structural comparisons reveal evolutionary relationships that are hidden in the 'twilight zone' [10] (less than $25 \%$ sequence identity) of sequence alignments.

We used the SCOP [11] database (version 1.32, representing all 4432 structures in the Protein Data Bank available up to May 1996) to identify possible more distant structural relatives of PCP. To compare with the N-terminal domain of PCP (153 residues, 8 helices), we used the 71 all- $\alpha$ folds enumerated in SCOP as possible candidates for structural similarity with PCP. From each of these folds, representatives were taken from a non-redundant database [12], superimposed [13] (Table 1) on the Nterminal domain of PCP and then compared visually using ' $\mathrm{O}$ ' [14]. Visual inspection was performed as the scores which are normally used for an automatic determination of topological similarity [13] were deemed inadequate for the identification of remote structural homology. Surprisingly, the topological similarity score [13] (column 5 in Table 1) matched well the visual finding that proteins of the globin-like fold (myoglobin, hemoglobin, phycobiliproteins and colicin A [15]) are structurally related to PCP (Fig. 2). In the case of the heme-binding globins, further support for the topological agreement found comes from overlapping tetrapyrrole binding sites, which are located on helices $\mathrm{E}$ and $\mathrm{F}$. The other all- $\alpha$ folds with a high topological similarity score (Table 1) are much larger proteins (252 to 605 residues) with some of their helices matching those of PCP by chance. Granulocyte-colony stimulating factor, a helix-bundle protein of similar size, has only 4 helices superimposable on PCP.
Table 1: Top-scoring all- $\alpha$ candidates for possible similarity to PCP. The all- $\alpha$ entries [11] from a nonredundant set [12] representing all known protein structures up to May 1996 were compared in an improved version of SUPERIMPOSE [13] with the N-terminal domain of PCP (153 residues).

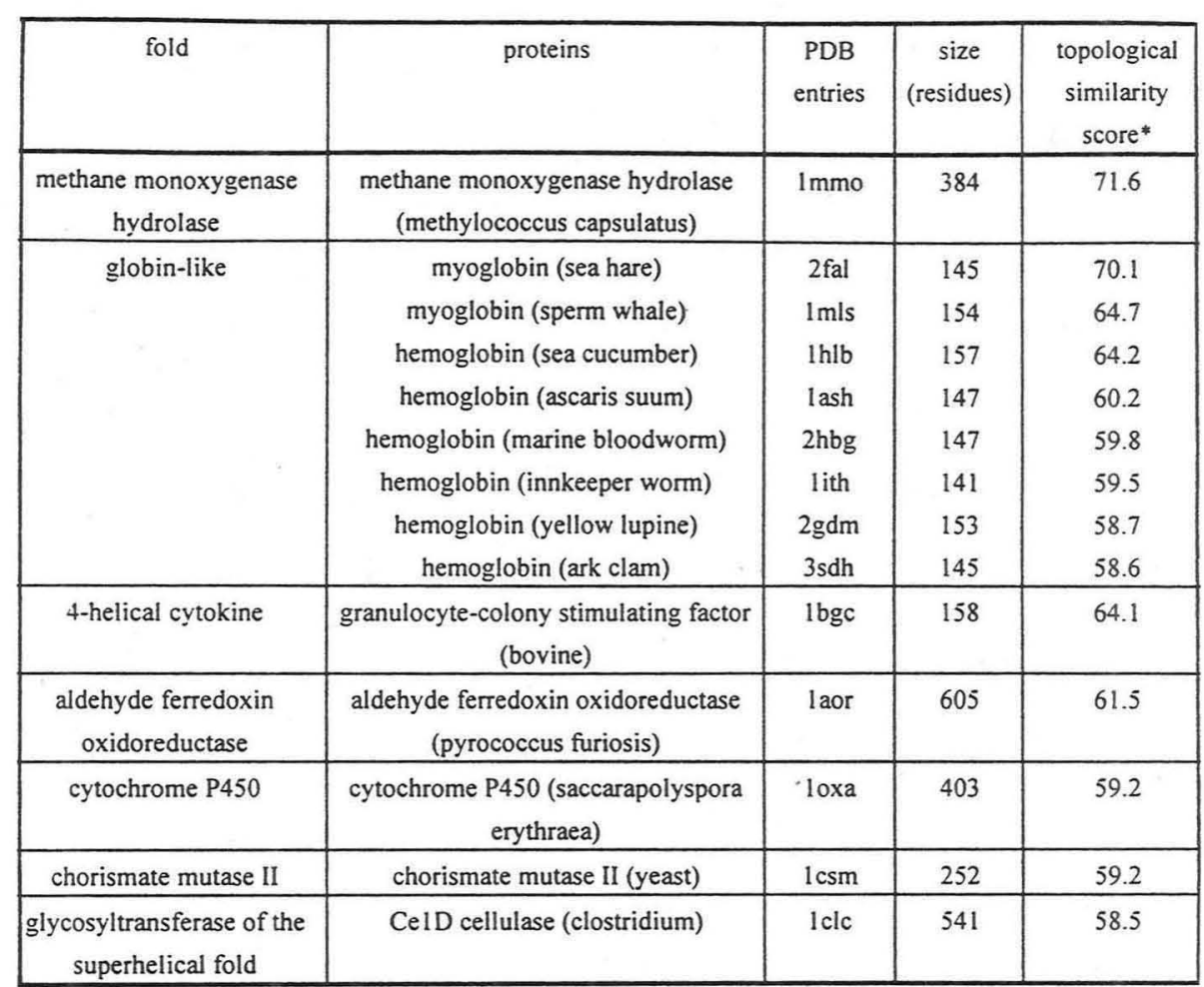

* for definition, see reference 13 .

It is worth discussing that the globin-fold has often been described as 3 helices (A,E,F) sandwiching at right angles on a layer of 3 other helices $(B, G, H)$. This ' 3 -on-3' description was found adequate for demonstrating structural relatedness of colicin A and globins [15], as well as that of phycobiliproteins and globins [16]. However, an early review of protein folding patterns [8] gave an alternative descriptions of the globin fold, the 'greek key' (so called according to its resemblance with decorative patterns on greek vases). We note that the greek key description is more informative, as it focuses on the connections between the helices inasmuch as on their packing, whereas the '3-on-3' term merely refers to the arrangement of helices in space, neglecting possibly differing topologies caused by different connections. In our first description of PCP [1], we identified its fold as a 'jellyroll', which is a special case of a greek key fold. However, a 3 -on-3 helical representation of PCP would, given its resemblance with the 
hull of a ship with helices A, F, E on one side and C, G, H on the other, therefore be possible as well. On the other hand, the angle between these 'sandwich layers' is closer to $0^{\circ}$ than to $90^{\circ}$ which is why we favour the greek key description of the PCP/globin fold. In Fig. 2, we therefore compare the folds of globins (in this case hemoglobin of ark clam) and PCP. As can be seen, both structures can be represented by nearly identical greek key topology diagrams (Fig. 2).

Other proteins exhibiting a globin-like fold and binding a related cofactor are the phycobiliproteins. While these LHCs are functionally closely related to PCP, they do not structurally agree better with PCP's N-terminal domain than do globins (r.m.s.d. $=4.8 \AA$ for phycocyanin [17] $v s$. approx. $4.3 \AA$ for globins, superimposing about 100 residues). However, the fact that a very similar function is displayed by structurally related proteins makes a very strong point towards their phylogenetic relationship. In further support of this view, phycobiliproteins have developed an $\alpha$ - and a $\beta$-subunit of around 162 and 172 amino acids, respectively, which are quite similar in structure (r.m.s.d. $=1.3 \AA$ for phycocyanin), matching the development of long variant forms of PCP with closely related $\mathrm{N}$ - and C-terminal domains (r.m.s.d. $=0.9 \AA[1]$ ).

Figure 3 shows a superimposition of the three different tetrapyrrole cofactors of $\mathrm{PCP}$, myoglobin and phycocyanin. We find that the binding position is between the $\mathrm{E}$ and $\mathrm{F}$ helices in all three cases. However, the binding mode varies from buried in the case of PCP, to exposed in the case of the phycobiliproteins. Obviously, the key to the observation of structural deviations between PCP and phycobiliproteins lies in the different chromophores: whereas PCP provides a wide open pocket for a large number of hydrophobic pigments, the phycobiliproteins bind their extended open-chain tetrapyrroles on the outside of a compact apoprotein, with the globins occupying an intermediate position.

Based on these observations, we propose that PCP and the phycobiliproteins derive from a globin-like ancestor, and that the observed structural differences are largely due to the adaptation to different cofactors during divergent evolutionary pathways.

\section{How does the cargo get on board?}

Little is known about the folding pathway of most proteins. The general understanding is that folding from an extended state initiates at one or more places of the sequence in so-called 'folding nuclei', leading to a partial condensation of secondary structure elements which finally, driven by minimization of the free energy, collapse to the native state in a cooperative fashion. However, in the case of the globins, new methods (stopped-flow circular dichroism, hydrogen-exchange pulse labeling and temperaturejump fluorescence) for the study of folding intermediates in the 15 -ns to 5 -ms time scale have been developed and applied [18,19], resulting in a more detailed description of the folding pathway than available for any other family of proteins.
The emerging picture is that of a 'molten globule' state as an early protein-folding intermediate, with the A,G, and $\mathrm{H}$ helices forming the first native-like contacts. Using the topology diagrams (Fig. 2) for comparison, this means that condensation starts at the center of the greek key for proteins of the globin fold. In analogy to the globins, we therefore expect PCP to fold its A, G and $\mathrm{H}$ helices first. As the only substantial openings of the PCP monomer, those harbouring the lipid and the conjugated systems of the carotenoids (Fig. 1), are lined by the $\mathrm{H}$ and $\mathrm{G}$ helices, we postulate that these cofactors must be present during the first folding steps. It appears likely that the remaining steps during folding resemble those of the globins: addition of the $\mathrm{B}$ helix (C helix in PCP), and then the remaining F and E helices, thus completing the greek key. In the case of PCP, these steps are probably facilitated and guided by the presence of and the interaction with the cargo. As a result, the polypeptide chain wraps around the cofactors. We note that this condensation can happen simultaneously in the $\mathrm{N}$ - and Cterminal domains as the last helix of the $\mathrm{N}$-terminal domain is connected by a flexible linker of 13 residues (the 'keel', see Fig. 1) to the first helix of the C-terminal domain. Thus, the packing of the $\mathrm{N}$ - against the $\mathrm{C}$ - terminal carotenoids can be optimized by their interaction, and is not constrained by a rigid scaffold of the polypeptide.

\section{Future prospects}

Electrospray ionization-mass spectrometry has been shown [20] to be capable of observing intact protein molecules as well as identifying noncovalently attached cofactors. In collaboration with the group of M. Przybylski at the University of Konstanz, we are currently investigating in parallel the unfolding of myoglobin and PCP under native conditions and under mild proteolysis. Unfolding experiments can yield accurate information about the last steps of the folding process which are under consideration here, and we expect them to be compatible with our folding hypothesis outlined above. By detecting unfolding intermediates of PCP and its constituents, we especially hope to clarify the role of the lipids, which are integral components of the holoprotein with completely unknown function.

Acknowledgements: We thank Dr. M. Glocker for discussions.

Abbreviations:

PCP - Peridinin Chlorophyll $a$ Protein

LHC - Light Harvesting Complex

r.m.s.d. - root mean square deviation 


\section{References}

1 E. Hofmann, P.M. Wrench, F.P. Sharples, R.G. Hiller, W. Welte, and K. Diederichs (1996) Structural basis of light harvesting by carotenoids: peridinin-chlorophyll-protein from Amphidinium carterae. Science 272, 1788-1791.

2 G. McDermott, S.M. Prince, A.A. Freer, A.M. Hawthornthwaite-Lawless, M.Z. Papiz, R.J. Cogdell, and N.W. Isaacs (1995) Crystal structure of an integral membrane light-harvesting complex from photosynthetic bacteria. Nature 374, 517-521.

3 W. Kühlbrandt. D.N. Wang, and Y. Fujiyoshi (1994) Atomic model of plant light-harvesting complex by electron crystallography. Nature 367, 614-621.

4 N.S. Govind. S.J. Roman, R. Iglesias-Prieto, R.K. Trench, E.L. Triplett, and B.B. Prézelin (1990) An analysis of the light-harvesting peridinin-chlorophyll a-proteins from dinoflagellates by immunoblotting techniques. Proc. R. Soc. Lond. B240, 187-195.

5 Larkum, T. (1996) How dinoflagellates make light work with peridinin. Trends in Plant Science 8, 247248.

6 P.J. Kraulis (1991) MOLSCRIPT: a program to produce both detailed and schematic plots of protein structures. J. Appl. Cryst. 24, 946-950.

7 E.A. Merritt and M.E.P. Murphy (1994) RASTER3D version 2.0 - a program for photorealistic molecular graphics. A. Crystallogr. D50, 869-873.

8 J. Richardson (1981) The anatomy and taxonomy of protein structures. Adv. Prot. Chem. 34, 167-339.

9 L. Holm and C. Sander (1996) Mapping the protein universe. Science 273, 595-602.

10 U. Hobohm and C. Sander (1995) A sequence property approach to searching protein databases. J. Mol. Biol. 251, 390-399.

11 A.G. Murzin, S.E. Brenner, T. Hubbard, and C. Chothia (1995) Scop: a structural classification of proteins database for the investigation of sequences and structures. J.Mol.Biol. 247, 536-540.

12 U. Hobohm, M. Scharf, R. Schneider, and C. Sander (1992) Selection of a representative set of structures from the Brookhaven Protein Data Bank. Protein Science 1, 409-417.

13 K. Diederichs (1995) Structural superposition of proteins with unknown alignment and detection of topological similarity using a six-dimensional search algorithm. Proteins: Structure, Function and Genetics 23, 187-195.

14 T.A. Jones, J.Y. Zou, S.W. Cowan, and M. Kjeldgaard (1991) Improved methods for building protein models in electron density maps and the location of errors in these models. Acta Crystallogr. A47, 110119 .

15 L. Holm and C. Sander (1993) Structural alignment of globins, phycocyanins and colicin A. FEBS Letters 315, 301-306

16 T. Schirmer, W. Bode, R. Huber, W. Sidler, and H.J. Zuber (1985) X-ray crystallographic structure of the light-harvesting biliprotein C-phycocyanin from the thermophilic cyanobacterium Mastigocladus laminosus and its resemblance to globin structures. J. Mol. Biol. 184, 257-277.

17 M. Duerring G.B. Schmidt and R. Huber (1991) Isolation, crystallization, crystal structure analysis and refinement of constitutive $\mathrm{C}$-phycocyanin from the chromatically adapting cyanobacterium Fremyello diplosiphon at 1.66̊̊ resolution. J. Mol. Biol. 217, 577-592.

18 P.A. Jennings and P.E. Wright (1993) Formation of a molten globule intermediate early in the kinetic folding pathway of apomyogiobin. Science 262, 892-896.

19 R.M. Ballew, J. Sabelko, and M. Grueble (1996) Direct observation of fast protein folding: the initial collapse of apomyoglobin. Proc. Natl. Acad. Sci. USA 93, 5759-5764.

20 several articles in this volume 